\title{
Different Profiles of the Negatively Stained Citrus Canker Bacterium Xanthomonas citri pv. citri Depending on Culture Media and Heavy Metal Stains
}

\author{
Ki Woo Kim ${ }^{1 *}$, In Jung Lee 2 , Jae Wook Hyun ${ }^{3}$, Yong Hoon Lee ${ }^{4}$ and Eun Woo Park ${ }^{5 *}$ \\ ${ }^{1}$ National Instrumentation Center for Environmental Management, Seoul National University, Seoul 151-921, Korea \\ ${ }^{2}$ Research Institute for Agriculture and Life Sciences, Seoul National University, Seoul 151-921, Korea \\ ${ }^{3}$ Citrus Experiment Station, National Institute of Subtropical Agriculture, Rural Development Administration, Jeju 697-943, Korea \\ ${ }^{4}$ Department of Biotechnology, Chonbuk National University, Iksan 570-752, Korea \\ ${ }^{5}$ Department of Agricultural Biotechnology, Seoul National University, Seoul 151-921, Korea \\ (Received on September 1, 2009; Accepted on February 4, 2010)
}

\begin{abstract}
Staining profiles and bacterial morphology were compared in Xanthomonas citri pv. citri by a transmission electron microscopy. Four types of negative staining regimes were employed depending on culture media and heavy metal stains. The bacterial cells grown on $\mathrm{LB}$ agar media often appeared clustered on the supporting film. Meanwhile, individual bacterial cells could be readily found on the preparations from $L B$ broth media. Typical rod-shaped cells (ca. $1 \mu \mathrm{m}$ in length) and their flagella were observed in either $2 \%$ uranyl acetate (UA) or $2 \%$ neutralized potassium phosphotungstate (PTA) staining. The UA-stained bacteria often showed relatively intact cell morphology and rather positively stained cells with a thin electron-dense stain depth around bacteria. The PTA-stained bacteria were characterized by the wrinkled cell surface where the stain was entrapped in grooves. In addition, distinct electron-dense stain depth was evident around the PTA-stained preparations. Numerous fimbriae could be mostly observed from the PTA-stained preparations of the two culture media, but not from the UA-stained preparations.
\end{abstract}

Keywords : citrus canker, fimbriae, flagella, negative staining, Xanthomonas citri pv. citri.

Negative staining is a simple and rapid method in transmission electron microscopy to observe the morphology and structure of particulate specimens such as bacteria, viruses, cell components, and isolated macromolecules (Hayat, 2000). The staining is usually achieved by heavy metal stain solutions which occupy hydrated regions around and within the objects, and form a cast upon drying onto a

\footnotetext{
*Co-Corresponding authors.

KW Kim

Phone) +82-2-880-4957, FAX) +82-2-888-4847

E-mail)kiwoo@snu.ac.kr

EW Park

Phone) +82-2-880-4672, FAX) +82-2-873-2317

E-mail)ewpark@snu.ac.kr
}

thin supporting film (Hoppert and Holzenburg, 1998). Ideally, negative stains should not bind to the objects which are represented by stain-excluding areas, that is, they remain electron-translucent in transmission electron microscopy (Hoppert and Holzenburg, 1998). However, a certain degree of interaction between charged groups of the specimen and staining ions may occur and produce unwanted positive staining (Hayat, 2000).

The two commonly used heavy metal stains for negative staining include uranyl acetate $\left(\mathrm{UA} ; \mathrm{C}_{4} \mathrm{H}_{6} \mathrm{O}_{6} \mathrm{U}\right)$ and neutralized potassium (or sodium) phosphotungstate (PTA; $\mathrm{H}_{3} \mathrm{PW}_{12} \mathrm{O}_{40}$ ). UA is a typical cationic heavy metal salt, whereas PTA is a traditional anionic heavy metal salt. It is known that the uranyl cation $\left(\mathrm{UO}_{2}^{2+}\right)$ of $\mathrm{UA}$ at a $\mathrm{pH}$ of approximately 4.5 has a net positive charge and reacts strongly with phosphate and amino groups (Bozzola and Russell, 1999; Hayat, 2000). At a neutral pH, the phosphotungstic anion $\left(\mathrm{PW}_{12} \mathrm{O}_{40}{ }^{3-}\right)$ of PTA has a net negative charge and is repelled by the negative charge of most proteins (Hayat, 2000).

The Xanthomonas citri pv. citri (syn. X. axonopodis pv. citri) is a pathogen of the Asiatic type of citrus canker (Schaad et al., 2005). As a gram negative bacterium, the pathogen has flagella and type IV fimbriae (Moreira et al., 2004). Fimbriae of plant pathogenic bacteria are involved in various functions: biofilm formation on the host surface, attachment to stomata and hydathodes, and twitching motility (Van Doorn et al., 2001). However, little information is available on the factors governing the visualization of fimbriae of $X$. citri pv. citri. Although it is commonly assumed that either culture conditions or negative stains could be insignificant in revealing bacterial morphology, the corresponding different staining profiles would provide any incorrect or misleading observations, leading to a different hypothesis. Therefore, it is crucial to unravel the effects of different culture media and negative stains on the visualization of bacterial appendages for a comprehensive understanding of the bacterial movement, adhesion, and pathogenicity. The objectives of this study were to i) de- 
monstrate the versatility of negative staining profiles of $X$. citri pv. citri according to culture conditions and metal stains, and ii) propose an optimal condition for the visualization of the bacterial appendages in the context of routine applications.

Bacterial strain. A strain $(\mathrm{kxj} 31)$ of $X$. citri pv. citri was prepared as reported previously (Lee et al., 2009). The strain was subcultured from a frozen stock culture and grown on LB agar medium to ensure its viability.

Negative staining regimes. To determine the effects of culture media and heavy metal stains on staining profiles of the pathogen, four types of negative staining regimes were employed as follows: (i) 2\% (w/v) UA staining after growing on LB agar medium (solid medium/UA), (ii) $2 \%$ (w/v) PTA ( $\mathrm{pH} 7.0)$ staining after growing on LB agar medium (solid medium/PTA), (iii) 2\% UA staining after growing on LB broth medium (liquid medium/UA), and (iv) 2\% PTA staining after growing on LB broth medium (liquid medium/ PTA). One colony grown on $\mathrm{LB}$ agar medium at $30^{\circ} \mathrm{C}$ for one day was gently suspended in one $\mathrm{ml}$ of triple distilled water (Kim et al., 2008). In addition, the bacteria grown in $\mathrm{LB}$ broth medium at $30^{\circ} \mathrm{C}$ for $12 \mathrm{~h}$ were diluted with triple

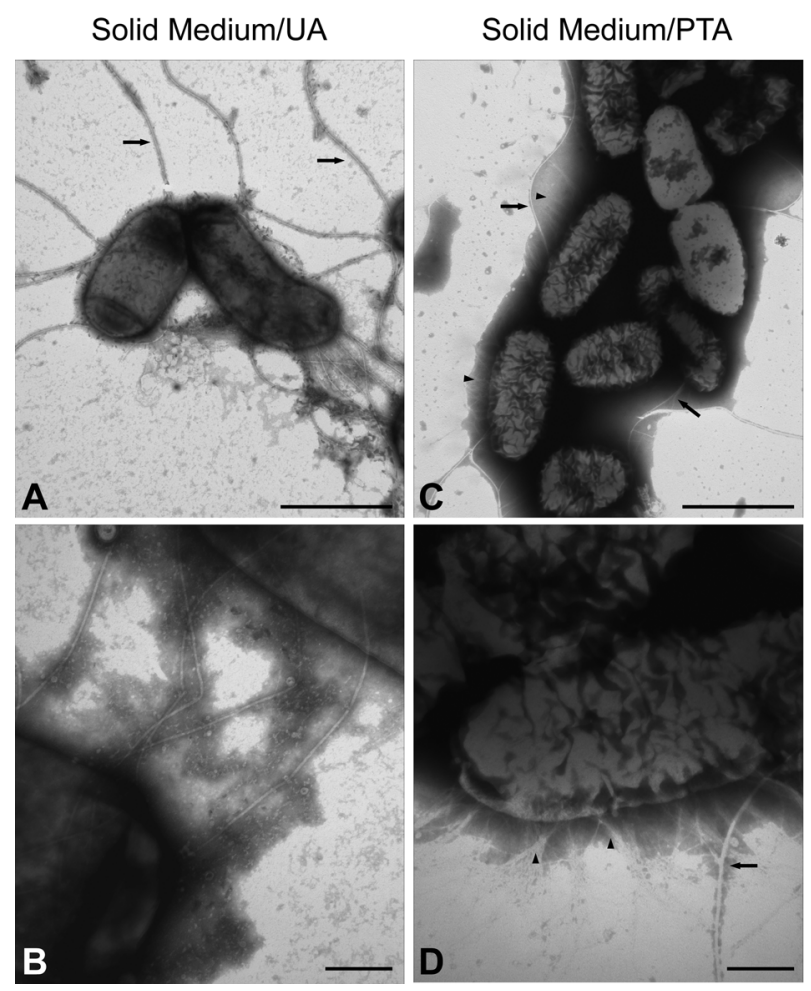

Fig. 1. Transmission electron micrographs of $X$. citri pv. citri grown on LB agar media. (A and B) UA-stained bacteria. Arrows indicate flagella. (C and D) PTA-stained bacteria. Flagella (arrows) and fimbriae (arrowheads) were noted. Bars in A and $\mathrm{C}$ $=1 \mu \mathrm{m}$. Bars in $\mathrm{B}$ and $\mathrm{D}=200 \mathrm{~nm}$. distilled water for vaguely observable turbidity. A drop (ca. $10 \mu \mathrm{l}$ ) of each bacterial suspension was adsorbed to a glowdischarged formvar-coated copper grid for $1 \mathrm{~min}$. The excess liquid was drained off with filter paper, and the preparations were stained with either $2 \%$ UA or $2 \%$ PTA for 10 seconds, washed briefly with two drops of triple distilled water, and air-dried. The specimens were examined with a transmission electron microscope (JEM-1010; JEOL, Tokyo, Japan) operated at an accelerating voltage of $80 \mathrm{kV}$.

Staining profiles and bacterial morphology. There were differences in the bacterial morphology and staining profiles of $X$. citri $\mathrm{pv}$. citri among the negative staining regimes. The bacterial cells grown on the solid medium often appeared clustered on the supporting film. Typical rod-shaped cells (ca. $1 \mu \mathrm{m}$ in length) and their flagella were observed in the UA staining after growing on the solid medium (Fig. 1A). Higher magnifications clearly showed a thin electrondense rim around bacteria (Fig. 1B). Stain depth was evident around the PTA-stained bacteria after growing on the solid medium (Fig. 1C). Furthermore, numerous fimbriae could be observed around bacteria at higher magnifications (Fig.

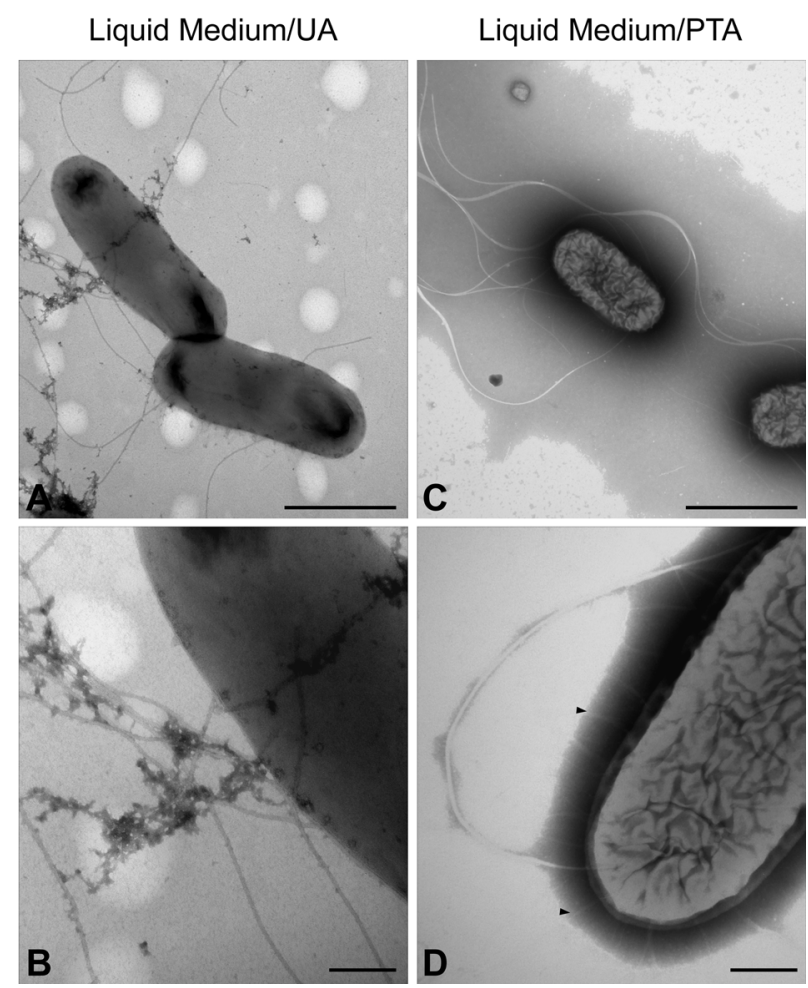

Fig. 2. Transmission electron micrographs of $X$. citri pv. citri grown in LB broth medium. (A and B) UA-stained bacteria. Fimbriae could be hardly found around bacteria. Electron-dense precipitates possibly from the medium were often found on the flagella. (C and D) PTA-stained bacteria. Numerous fimbriae (arrowheads) were observed. Bars in $\mathrm{A}$ and $\mathrm{C}=1 \mu \mathrm{m}$. Bars in $\mathrm{B}$ and $\mathrm{D}=200 \mathrm{~nm}$. 
1D).

It was common to find $X$. citri $\mathrm{pv}$. citri cells evenly spread on the supporting film and rather positively stained with UA after growing in the liquid medium (Fig. 2A). Neither distinct stain depth nor fimbriae could be discernable around the UA-stained bacteria even at higher magnifications (Fig. 2B). Meanwhile, the PTA-stained bacteria after growing in the liquid medium had distinct electron-dense stain depth (Fig. 2C). Flagella and fimbriae were usually contrasted with electron-dense stain depth around bacteria against background (Fig. 2D).

This study enhanced our knowledge of the negative staining of $X$. citri pv. citri and provided experimental precautions to be considered for routine applications. The culture media affected the staining profiles and bacterial morphology in this study. In spite of the pre-stain suspension to remove most of the extraneous materials, the clustering of bacteria grown on the solid medium could not be completely avoided, possibly owing to the extracellular polysaccharides. Meanwhile, individual bacterial cells could be readily found on the preparations from the liquid culture. Different staining profiles and bacterial morphology were also evident depending on the negative stains. Due to their different chemical features including electric charges, the two stains have different contrast ability, leading to different staining profiles of the specimen (Ohi et al., 2004; Walz et al., 1995). Bacteria have surfaces that are reactive with metals largely due to the dissociation of protons from carboxyl and phosphoryl group constituents of cell wall macromolecules (Glasauer et al., 2001). Largely due to the negatively charged bacterial cell surface, the bacteria were not intensively positively stained by PTA. The PTA-stained bacteria were also characterized by the wrinkled cell surface where the stain was entrapped in grooves (Hoppert and Holzenburg, 1998). The UA-stained bacteria, however, often showed relatively intact cell morphology, because UA stabilized lipids as a fixative and thus minimized the deleterious effect of drying on the specimen (Hayat, 2000).

An intriguing finding was that fimbriae could be mostly observed from the PTA-stained preparations of either the solid or liquid medium, but not from the UA-stained preparations. These data suggest that an appropriate negative stain should be chosen depending on the research objectives and the cell structures of interest. In particular, it was proposed from this study that PTA staining could be better than UA staining to visualize both flagella and fimbriae of $X$. citri pv. citri from either the solid or liquid medium. The application of proper culture media and heavy metal stains for negative staining will facilitate morphological characterization of diverse isolates of $X$. citri pv. citri with different levels of surface attachment to hosts.

\section{Acknowledgments}

This study was supported by the Agricultural Research \& Development Promotion Center (Project No. 105058-3), Korea.

\section{References}

Bozzola, J. J. and Russell, L. D. 1999. Electron Microscopy: Principles and Techniques for Biologists, 2nd Ed. Jones and Bartlett Publishers, Boston, NY. 670 pp.

Glasauer, S., Langley, S. and Beveridge, T. J. 2001. Sorption of Fe (hydr)oxides to the surface of Shewanella putrefaciens: Cellbound fine-grained minerals are not always formed de novo. Appl. Environ. Microbiol. 67:5544-5550.

Hayat, M. A. 2000. Principles and Techniques of Electron Microscopy: Biological Applications, 4th Ed. Cambridge University Press, Cambridge, UK. 543 pp.

Hoppert, M. and Holzenburg, A. 1998. Electron Microscopy in Microbiology. BIOS Scientific Publishers, Oxford, UK. 112 pp.

Kim, K. W., Jung, W. K. and Park, Y. H. 2008. Comparison of pre-stain suspension liquids in the contrasting ability of neutralized potassium phosphotungstate for negative staining of bacteria. J. Microbiol. Biotech. 18:1762-1767.

Lee, I. J., Kim, K. W., Hyun, J. W., Lee, Y. H. and Park, E. W. 2009. Comparative ultrastructure of nonwounded Mexican lime and Yuzu leaves infected with the citrus canker bacterium Xanthomonas citri pv. citri. Microsc. Res. Tech. 72:507-516.

Moreira, L. M., De Souza, R. F., Almeida, N. F. Jr., Setubal, J. C., Oliveira, J. C. F., Furlan, L. R., Ferro, J. A. and Da Silva, A. C. R. 2004. Comparative genomics analyses of citrus-associated bacteria. Annu. Rev. Phytopathol. 42:163-184.

Ohi, M., Li, Y., Cheng, Y. and Walz, T. 2004. Negative staining and image classification-powerful tools in modern electron microscopy. Biol. Proced. Online 6:23-34.

Schaad, N. W., Postnikova, E., Lacy, G. H., Sechler, A., Agarkova, I., Stromberg, P. E., Stromberg, V. K. and Vidaver, A. K. 2005. Reclassification of Xanthomonas species pathogenic on citrus. Sys. Appl. Microbiol. 28:494-518.

Stone, K. D., Zhang, H.-Z., Carlson, L. K. and Donnenberg, M. S. 1996. A cluster of fourteen genes from enteropathogenic Escherichia coli is sufficient for the biogenesis of a type IV pilus. Mol. Microbiol. 20:325-337.

Van Doorn, J., Hollinger, T. C. and Oudega, B. 2001. Analysis of the type IV fimbrial-subunit gene fimA of Xanthomonas hyacinthi: Application in PCR-mediated detection of yellow disease in hyacinths. Appl. Environ. Microbiol. 67:598-607.

Walz, T., Häner, M., Wu, X.-R., Henn, C., Engel, A., Sun, T.-T. and Aebi, U. 1995. Towards the molecular architecture of the asymmetric unit membrane of the mammalian urinary bladder epithelium: a closed "twisted ribbon" structure. J. Mol. Biol. 248:887-900. 